\title{
DETECTION OF TRANSIENT SIGNALS BY WAVELET PACKET TRANSFORM AND STOCHASTIC RESONANCE
}

\author{
Aparna Dutta and G.V.Anand \\ Department of Electrical Communication Engineering \\ Indian Institute of Science, Bangalore-560 012, INDIA \\ Email: anandgv@ece.iisc.ernet.in
}

\begin{abstract}
The problem of detecting an unknown transient signal in noise is considered. The observed data is first quantized by a symmetric quantizer. By an optimum matching of the quantizer thresholds to the noise probability density function, the signalto-noise ratio in the data is enhanced by the phenomenon of stochastic resonance. The output of the stochastic resonator(optimal quantizer) is then transformed using a translation-invariant wavelet packet transform (TIWPT). The magnitude of the maximum magnitude coefficient in the entire TIWPT decomposition is used as the test-statistic. Simulation results are presented to show that, at very low SNRs, the TIWPT detector preceded by the stochastic resonator gives a much better detection performance than a detector based on the TIWPT alone.
\end{abstract}

\section{INTRODUCTION}

Detection of transient signals embedded in noise is a problem of great interest in several applications such as passive detection of targets in the ocean, detection and localization of transients in ECG and EEG, and automatic machinery monitoring. Most transient detection techniques are based on the wavelet transform or the wavelet packet transfo rm[?, ?]. Recently, Ravier and Amblard[?] have proposed denoising using higher order statistics to improve the performance of the wavelet-packet based transient detector. In this paper, we present a method of using stochastic resonance to enhance the performance of transient detectors in non-Gaus sian noise. Such detectors would be of interest for transient detection in the ocean where the ambient noise is known to have non-Gaussian statistics. Stochastic resonance (SR) is the phenomenon of enhancement of signal transmission by certain non-linear systems resulting from the addition of noise to the system[?]. Quantizers and other static nonlinear systems exhibit SR [5,6,7]. driven by a deterministic signal and stationary white noise, the output signal-to-noise ratio (SNR) increases as the input noise variance $\sigma^{2}$ is increased over

0-7803-8560-8/04/\$20.00(C) 2004IEEE a certain range of values of $\sigma^{2}$. While conventional studies of SR maximize the output SNR by optimizing the input noise level, keeping the system fixed, in detection schemes the approach is reversed; the system parameters are optimized for a given noise level and type. The peak SNR gain of the SR system may exceed unity if the noise is non-Gaussian, and under these conditions the performance of a conventional detector may be improved by combining it with a stochastic resonator. It is known that, for the detection of a periodic signal in non-Gaussian noise, the combination of a stochastic resonator and matched filter yields a better detection performance than that of the matched filter alone $[6,7,8]$.

In this paper, we present a scheme for enhancing the performance of the transient detector based on a translation-invariant wavelet packet transform(TIWPT) by combining it with an optimal 3 -level quantizer. The discrete time signal $s_{1}[n]$ is modelled as an exponentially damped sinusoid

$$
\begin{aligned}
s_{1}[n]= & A \exp \left(-\alpha\left(n-n_{o}\right)\right)\left(1-\exp \left(-\beta\left(n-n_{o}\right)\right)\right) \\
& \cdot \cos \left(2 \pi f\left(n-n_{o}\right)+\phi\right) u\left[n-n_{o}\right],
\end{aligned}
$$

with unknown amplitude $A$, frequency $f$, phase $\phi$, decay rate $\alpha$, rise rate $\beta$ and arrival time $n_{o}$. The transient nature of the signal, the low signal energy, and the presence of several unknown signal parameters render the matched filter or the GLRT based detector [9] unsuitable for detecting such signals, and hence the TIWPT detector is preferred. The noise is assumed to be zero-mean, independent and identically distributed(i.i.d) with generalized gaussian(GG) or mixture-of-Gaussian(MG) probability density function(PDF). The GG PDF of exponent $p>0$ and variance $\sigma^{2}$ is given by

$$
f(u)=a \exp \left(-|b u|^{p}\right),
$$

where $a=\frac{p}{2 \sigma} \Gamma^{\frac{1}{2}}\left(\frac{3}{p}\right) / \Gamma^{\frac{3}{2}}\left(\frac{1}{p}\right)$ and $\left.b=\frac{1}{\sigma}\left\{\Gamma^{\left(\frac{3}{p}\right)}\right) / \Gamma\left(\frac{1}{p}\right)\right\}^{\frac{1}{2}}$. The MG PDF with parameters $c \in(0,1), d_{1}>$ $0, d_{2}>0$ is given by

$f(u)=\frac{c}{d_{1} \sqrt{2 \pi}} \exp \left(\frac{-u^{2}}{2 d_{1}^{2}}\right)+\frac{1-c}{d_{2} \sqrt{2 \pi}} \exp \left(\frac{-u^{2}}{2 d_{2}^{2}}\right)$.

These PDFs are chosen since they provide good models for a wide variety of noise environments in 
the ocean[10]. The theory of transient detection by TIWPT[?] is reviewed in Section 2. The optimal design of 3-level quantizers is discussed in Section 3. Design of a transient detector based on SR and TIWPT is presented in Section 4, along with simulation results. It is shown that, for a large class of GG and MG noise PDFs, the performance of the SR-TIWPT detector is significantly better than that of the detector based on TIWPT alone.

\section{TRANSIENT DETECTION BY TIWPT}

Consider a data vector $\mathbf{x}=\left[\begin{array}{ll}x[1] & x[2] \ldots x[N]\end{array}\right]^{T}$, where $N=2^{J}$ and $J$ is a positive integer. $\mathbf{x}=$ $\mathbf{s}_{1}+\sigma \mathbf{w}$ if the signal is present and $\mathbf{x}=\sigma \mathbf{w}$ otherwise, where $\mathbf{s}_{1}$ is the transient signal and $\sigma \mathbf{w}$ is i.i.d noise with mean 0 and variance $\sigma^{2}$. Consider a full ( $J$-level) wavelet packet transform(WPT) of $\mathbf{x}$ and let $\mathcal{C}$ be the set of WPT coefficients of all levels from 1 to $J$. Since the transient signal is confined to a small region of support in the timefrequency plane, the signal energy is concentrated in a small number of relatively large coefficients in the set $\mathcal{C}$. On the other hand, the average energy of i.i.d. noise is uniformly distributed over all WPT coefficients since WPT is an orthogonal transform. Hence, the magnitude of the maximum magnitude coefficient in the set $\mathcal{C}$ may be used as the teststatistic $T(\mathbf{x})$ for signal detection along with the following decision rule: decide signal present if $T(\mathbf{x})>\eta$, decide signal absent otherwise. The threshold $\eta$ determines the probability of detection $P_{D}$ and the probability of false alarm $P_{F A}$. The detector performance is characterized by the receiver operating characteristic(ROC), which is a plot of $P_{D}$ vs $P_{F A}$.

Due to the downsampling inherent in the WPT, the set $\mathcal{C}$ of WPT coefficients changes if the signal is shifted in time by an odd number of samples. Hence $T(\mathbf{x})$ changes if the signal is shifted in time, which is not a desirable characteristic of a detector. Translation invariance(TI) of the WPT can be achieved in the following manner[?]. Let the unit circular shift operator $S_{m}$ operating on $\mathbf{y} \in \mathcal{R}^{m}$ be defined as $S_{m}(y[n])=y[(n+1) \bmod m]$. Let the operator $F_{m}$ defined on $\mathcal{R}^{m}$ be the one-level WPT operator, i.e, $F_{m}(\mathbf{y}), \mathbf{y} \in \mathcal{R}^{m}$, gives two sequences of length $m / 2$. The full WPT consists of recursive applications of $F_{m}$ with $m=2^{j}, j=J, J-1, \ldots, 1$. Thus the input signal $\mathbf{x}$ of length $2^{J}$ is transformed into two sequences of length $2^{J-1}$, then four sequences of length $2^{J-2}$, and so on. At each level of decomposition, we maximise an objective function $M(\mathbf{y})$ defined as

$$
M(\mathbf{y})=\max (|y[0]|,|y[1]|, \ldots,|y[m-1]|), \mathbf{y} \in \mathcal{R}^{m}
$$
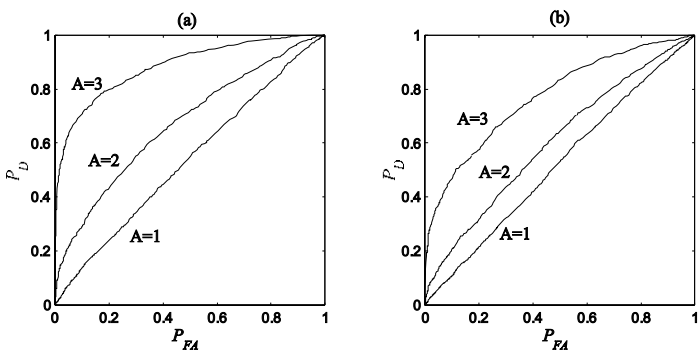

Fig. 1. ROCs for different amplitude signals for two different noise PDFs (a) GG noise with $p=8$ and (b) MG noise with $c=0.2$ and $d_{2} / d_{1}=10$

The TIWPT is defined as follows:

$F_{m}^{T I}(\mathbf{y})= \begin{cases}F_{m}(\mathbf{y}) & \text { if } M\left(F_{m}(\mathbf{y})\right)=M_{\max } \\ F_{m}\left(S_{m}(\mathbf{y})\right) & \text { if } M\left(F_{m}\left(S_{m}(\mathbf{y})\right)\right)=M_{\max }\end{cases}$

where $M_{\max }=\max \left\{M\left(F_{m}(\mathbf{y})\right), M\left(F_{m}\left(S_{m}(\mathbf{y})\right)\right)\right\}$. Thus TIWPT calculates, at each level and node of the decomposition, the absolute maximum coefficient of the decomposition of the unshifted sequence and that of the sequence circularly shifted by one. The sequence with the larger absolute maximum coefficient is chosen. Once the TIWPT coefficients are chosen in this way, the magnitude of the largest magnitude coefficient in the entire set of coefficients is used as the test-statistic $T(\mathbf{x})$.

The ROCs of the TIWPT detector are plotted in Fig ?? for two different noise PDFs and different values of the signal amplitude $A$. The aim of the present work is to improve the detection performance, especially at low signal amplitudes.

\section{SNR ENHANCEMENT OF NOISY SIGNALS USING STOCHASTIC RESONANCE}

Consider a non-linear memoryless system with input

$$
x[n]=s_{1}[n]+\sigma w[n], n=1,2, \ldots, N,
$$

where $s_{1}[n]$ is the signal and $\sigma w[n]$ is iid noise with mean 0 and variance $\sigma^{2}$. Let the output of the system be denoted by $y[n]$. The SNRs at the input and the output of the system are defined as

$$
\begin{gathered}
S N R_{i n}=\frac{\sum_{n=1}^{N} s_{1}^{2}[n]}{\sigma^{2}}, \\
S N R_{o u t}=\frac{\left(\sum_{n=1}^{N} s_{1}[n] E\{y[n]\}\right)^{2}}{\sum_{n=1}^{N} s_{1}^{2}[n] \frac{1}{N} \sum_{n=1}^{N} \operatorname{var}\{y[n]\}} .
\end{gathered}
$$

The SNR gain, defined as the ratio of the output SNR to the input SNR, is given by

$$
G=\frac{\left(\sum_{n=1}^{N} s[n] E\{y[n]\}\right)^{2}}{\frac{1}{N} \sum_{n=1}^{N} \operatorname{var}\{y[n]\}\left(\sum_{n=1}^{N} s^{2}[n]\right)^{2}},
$$

where $s[n]=s_{1}[n] / \sigma$. 
SR systems such as the 3-level quantizer described below can be tuned(optimized) to yield SNR gain greater than unity. Such systems may be used to boost the SNR of the observed data for a better detection performance as shown in Fig ??. Let the data sequence $\{x[n], n=1,2, \ldots, N\}$ be

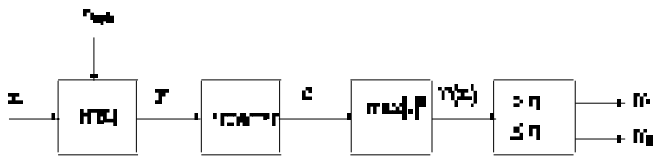

Fig. 2. Detection scheme using SR and TIWP transform

the input to a symmetric 3-level quantizer whose input-output relation is given by

$$
y[n]=\left\{\begin{array}{lll}
-1 & \text { for } & x[n] \leq-\gamma_{1} \\
0 & \text { for } & -\gamma_{1}<x[n] \leq \gamma_{1} . \\
1 & \text { for } & x[n]>\gamma_{1} .
\end{array}\right.
$$

It can be readily shown that, if the quantizer input contains the signal, the mean and variance of the output are given by

$$
\begin{aligned}
E\{y[n]\} & =F(\gamma+s[n])-F(\gamma-s[n]) \\
E\left\{y^{2}[n]\right\} & =2-F(\gamma+s[n])-F(\gamma-s[n] 01 \\
\operatorname{var}\{y[n]\} & =E\left\{y^{2}[n]\right\}-E^{2}\{y[n]\}
\end{aligned}
$$

where $\gamma=\gamma_{1} / \sigma$, and $\boldsymbol{F}($.$) denotes the cumula-$ tive distribution function of the unit-variance noise random variable $w[n]$. The SNR gain $G$ can be determined by substituting $\mathrm{Eq}(? ?)$ and $\mathrm{Eq}(? ?)$ into $\mathrm{Eq}(? ?)$.

It is evident that $G$ depends on the quantizer threshold, the signal, and the noise PDF. Since the signal detection performance improves as the SNR increases, it is of interest to determine the value of $\gamma$ for which $G$ is maximum by finding the real roots of the equation $\frac{\partial G}{\partial \gamma}=0$. The optimal value of $\gamma$ is denoted by $\gamma_{o p t}$ and the optimal value of the threshold $\gamma_{1}$ is $\sigma \gamma_{o p t}$. The maximum SNR gain is denoted by $G_{S R}$. Both $\gamma_{o p t}$ and $G_{S R}$ depend on the noise PDF and the signal. The quantizer that employs the optimal threshold is called the SR Quantizer(SRQ) or the stochastic resonator.

We have done extensive computations to investigate the dependence of $G_{S R}$ and $\gamma_{o p t}$ on various signal parameters. The variation of $G_{S R}$ and $\gamma_{o p t}$ with the signal amplitude $A$ and decay rate $\alpha$ is shown in Fig ?? for noise PDFs belonging to the GG family. For all these curves, values of the signal parameters (when not being varied) are $f=0.1$ cycles $/$ sample, $\phi=0$ radians, $A=1.5$, $\alpha=0.1 /$ sample and $\beta=0.5 /$ sample. Our computations indicate that the dependence of $G_{S R}$ and $\gamma_{o p t}$ on other signal parameters is generally quite weak. One noteworthy observation is that $G_{S R}$ never exceeds unity if the noise is Gaussian (i.e, $p=2$ for the GG family and $d_{2} / d_{1}=1$ for the MG family). One convenient measure of nonGaussianity of a zero-mean random variable is its kurtosis $\kappa$ defined as $\kappa=E\left(x^{4}[n]\right) / E^{2}\left(x^{2}[n]\right)-3$. For the Gaussian pdf, $\kappa=0$. For a non-Gaussian PDF, $\kappa$ may be either positive or negative. The kurtosis of a GG PDF of exponent $p$ is given by
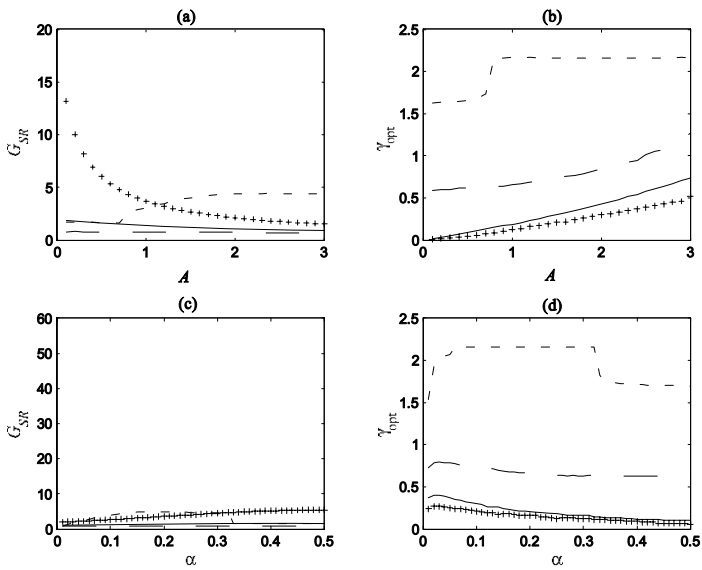

Fig. 3. Variation of $G_{S R}$ and $\gamma_{\text {opt }}$ with signal parameters $A$ and $\alpha$ for GG noise of exponent $0.5(+)$, $1(-), 2(--), 8(-\cdot)$ and $30(\cdot)$

$$
\kappa=3\left(c d_{1}^{4}+(1-c) d_{2}^{4}\right)-3 .
$$

Plots of $G_{S R}$ against the kurtosis $\kappa$ are shown in Fig ?? for GG and MG noise PDFs. It is seen that $G_{S R}$ increases as $|\kappa|$ is increased, i.e, progressively higher gains are achievable as the degree of nonGaussianity increases.
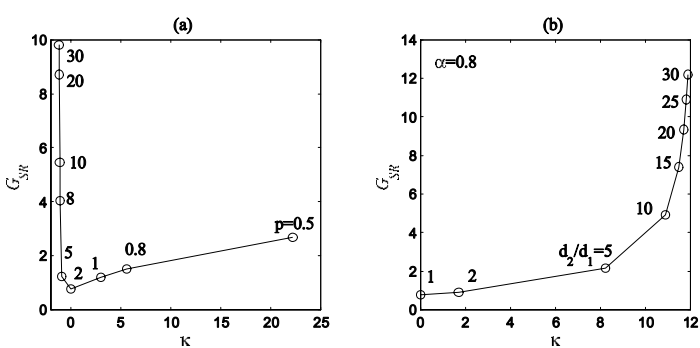

Fig. 4. Variation of $G_{S R}$ with kurtosis $\kappa$ for (a)GG noise and (b) MG noise with $c=0.8$ for $s[n]=1.5 \exp (-0.1 n)[1-$ $\exp (-0.5 n)] \cos (2 \pi(0.1) n)$

\section{TRANSIENT DETECTION USING SR AND TIWPT}

The proposed transient detection scheme based on SR and TIWPT is shown in Fig ??. Since the SNR gain $G$ is dependent on the noise PDF as well as the signal, knowledge of the signal is necessary for maximising $G$. If the signal parameters are random, a knowledge of the PDFs of the signal parameters may be used to find the optimal threshold. Let $\mathbf{p}=\left[\begin{array}{lllll}A & f & \alpha & \beta & \phi\end{array}\right]$ be the vector 


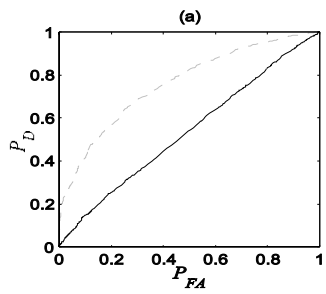

(c)

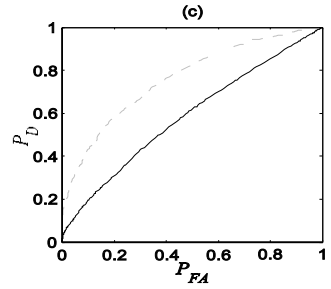

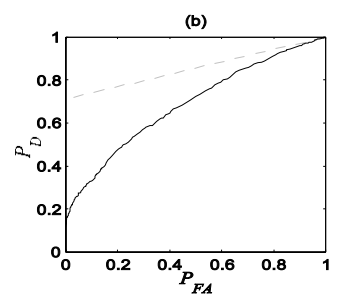

(d)

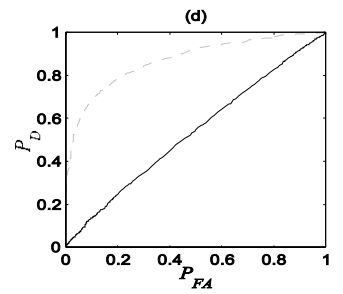

Fig. 5. ROCs for GG noise with (a) $p=0.5$ (b) $p=8$ and MG noise with (c) $c=0.5$ and $d_{2} / d_{1}=10$ and $(\mathrm{d}) c=0.8$ and $d_{2} / d_{1}=8$. Solid line : TIWPT, Dashed : 3-level SRQ+TIWPT

of signal parameters and let $f(\mathbf{p})$ be the PDF of p. Since $G=G(\mathbf{p}, \gamma)$, the expectation of $G$ for a given value of $\gamma$ can be written as

$$
E\{G \mid \gamma\}=\int_{\mathbf{p}} G(\mathbf{p}, \gamma) f(\mathbf{p}) d \mathbf{p} .
$$

$E\{G \mid \gamma\}$ is maximised with respect to $\gamma$ to obtain the optimal threshold $\gamma_{\text {opt }}$. ROCs of the TIWPT detector alone and the TIWPT detector preceded by a 3-level SRQ are plotted in Fig ?? for various noise PDFs. The number of observed samples was $N=256.1000$ different realizations each of noise and signal-plus-noise were used for simulations. For each realization of the transient, the parameters were chosen randomly as follows. $A / \sigma \sim$ $\mathcal{U}[1,3], \phi \sim \mathcal{U}[0,2 \pi] \mathrm{rad}, \alpha \sim \mathcal{U}[0.05,0.1]$ /sample, $f \sim \mathcal{U}[0.2,0.4]$ cycles/sample and $\beta=0.5 /$ sample (where $x \sim \mathcal{U}[a, b]$ means that $x$ is uniformly distributed between $a$ and $b$ ). The arrival time $n_{o}$ of the transient was uniformly randomly distributed between $-\frac{\ln 2}{\alpha}$ and $256-\frac{\ln 2}{\alpha}$ samples. This range of $n_{o}$ ensures that a signal that is spread across two frames is included in the simulation if the bulk of the signal energy is contained in the current frame. A 4-level decomposition was carried out using the Daubechies 4-tap(db4) wavelet filters. It was found that increasing the number of levels beyond four does not lead to any change in the test-statistic $T(\mathbf{x})$. It is seen from Fig ?? that the inclusion of the stochastic resonator improves the performance of the TIWPT detector significantly.

\section{CONCLUSION}

It has been shown that stochastic resonance in a 3level quantizer can be exploited effectively to bring about a significant improvement in the detection performance of a TIWPT detector of a transient signal immersed in noise for a wide range of nonGaussian noise PDFs.Work on application of this method to detection of transient acoustic signals transmitted through a shallow ocean is currently in progress.

\section{ACKNOWLEDGMENT}

This work was supported by the Defence Research and Development Organisation under the DRDOIISc Joint Programme on Advanced Research in Mathematical Engineering

\section{REFERENCES}

[1] M. Frisch and H. Messer, "The use of the Wavelet Transform in the Detection of an Unknown Transient Signal", IEEE Trans. Information Theory, Vol 38, pp 892-897,1992.

[2] S. D. Marco and J. Weiss, "Improved Transient Signal Detection Using a Wavepacket-Based Detector with an Extended Translation-Invariant Wavelet Transform", IEEE Trans.SP, Vol 45, No.4, pp 841-850,1997.

[3] P. Ravier and P. O. Amblard, "Wavelet Packets and De-noising based on HigherOrder Statistics for Transient Detection", Signal Processing, Vol 81, pp 19091926,2001.

[4] L. Gammaitoni, P. Hanggi, P. Jung, F. Marchesoni, "Stochastic Resonance", Rev. Modern Physics, Vol 70, pp 223287,1998 .

[5] F. Chapeau-Blondeau, "Stochastic Resonance and optimal detection of pulse trains by threshold devices", Digital Signal Processing, Vol 9, pp 162-177,1997.

[6] S.Zozor and P. O. Amblard, "On the use of Stochastic Resonance in Sine Detection", Signal Processing, Vol 82, pp 353367,2002 .

[7] A. A. Saha and G. V. Anand, "Design of detectors based on Stochastic Resonance", Signal Processing, Vol. 83, pp 1193-1212,2003.

[8] F. Chapeau-Blondeau, "Non-linear teststatistic to improve signal detection in non-Gaussian noise", IEEE Signal Processing Letters, Vol 7, pp 205-207,2000.

[9] S.M. Kay, Fundamentals of statistical signal processing, Vol II: Detection Theory, Chapters 4 and 7, Prentice Hall PTR, Upper Saddle River, New Jersey, 1998.

[10] E. J. Wegman, S. C. Schwartz, J. B. Thomas(ed), Topics in non-Gaussian Signal Processing, Chapters 2 and 3, Springer-Verlag,New York,1989. 